Technical Report No. 10/04, Nouvember 2004 ON A CLASSICAL RISK MODEL WITH A CONSTANT DIVIDEND BARRIER

Xiaowen Zhou 


\title{
On a classical risk model with a constant dividend barrier
}

\author{
Xiaowen Zhou * ${ }^{\dagger}$ \\ Department of Mathematics and Statistics, Concordia University, \\ Montreal H4B 1R6, Canada
}

\begin{abstract}
We consider a risk model with a constant dividend barrier. An explicit expression is obtained for the joint distribution of the surplus immediately prior to ruin and the deficit at ruin, discounted by the ruin time. Such an expression involves known results on the joint distribution at ruin for a classical risk model with single premium rate. The joint distributions related to the time periods when dividends are paid are also discussed. In particular, a new expression is obtained for the expected present value of the total amount of dividend payments until ruin.

JEL classification: $\mathrm{C} 65 ; \mathrm{G} 22$
\end{abstract}

$M S C: 60 \mathrm{G} 51$

Keywords: Expected present value of total dividends until ruin; Joint distribution of the surplus involving ruin time; Risk model with a constant dividend barrier

\section{Introduction and Preliminary Results}

The following risk model with a constant dividend barrier is considered in this paper. Let $\left\{N_{t}\right\}$ be a Poisson process with intensity $\lambda$. Let $X_{i}, i=1,2, \ldots$, be i.i.d. positive random variables with a common density function $p$, a common distribution function $P$, and a finite mean $\mu$. $\left\{N_{t}\right\}$ and $\left\{X_{i}\right\}$ are independent. For $u \geq 0$ the surplus process is defined by

$$
U_{t}^{*}=u+\int_{0}^{t} c\left(U_{s}^{*}\right) d s-\sum_{i=1}^{N_{t}} X_{i},
$$

where

$$
c(x):= \begin{cases}c, & \text { for } x \leq b, \\ 0, & \text { for } x>b .\end{cases}
$$

*Corresponding author. Tel.:514-848-2424-3220; Fax: 514-848-2831.

E-mail address: zhou@alcor.concordia.ca.

†Supported by an NSERC operating grant. 
$c, b>0$ are constants.

In such a surplus process $u \geq 0$ represents the initial surplus. The claims are arriving according to $\left\{N_{t}\right\} . X_{i}$ represents the size of the i-th claim. $c(x)$ represents the rate at which the premium is collected when the current surplus is $x .\left\{U_{t}^{*}\right\}$ stands for a surplus process for a model in which the insurance company would pay a dividend at rate $c$ once the surplus reaches level $b$, and stop the payment when the next incoming claim brings the surplus down to below level $b$. Notice that ruin occurs with probability 1 in such a model.

Write

$$
U_{t}:=u+c t-\sum_{i=1}^{N_{t}} X_{i},
$$

Then $\left\{U_{t}\right\}$ is the surplus process in a classical risk model with a single premium rate. The evolution of $\left\{U_{t}^{*}\right\}$ can be intuitively described as follows. $\left\{U_{t}^{*}\right\}$ behaviors like $\left\{U_{t}\right\}$ between 0 and $b$. But whenever $\left\{U_{t}^{*}\right\}$ reaches level $b$ it stops growing and keeps it value at $b$ for an exponential time with mean $\lambda^{-1}$ until the next claim brings it to under $b$.

Set $T^{*}(u):=\inf \left\{t \geq 0: U_{t}^{*}<0\right\}$ and $\psi^{*}(u):=\mathbb{P}\left\{T^{*}(u)<\infty\right\} . T^{*}(u)$ is the so-called ruin time, and $\psi^{*}(u)$ is the probability of ever ruin given that the initial surplus is $u$. For $u, \delta, y \geq 0$, let

$$
W^{*}(u ; \delta, x, y) d x d y=\mathbb{E}\left[e^{-\delta T^{*}(u)} ; U_{T^{*}(u)-}^{*} \in d x,-U_{T^{*}(u)}^{*} \in d y\right], 0 \leq x<b,
$$

and

$$
W^{*}(u ; \delta, b, y) d x d y=\mathbb{E}\left[e^{-\delta T^{*}(u)} ; U_{T^{*}(u)-}^{*}=b,-U_{T^{*}(u)}^{*} \in d y\right]
$$

We can define $T(u), \psi(u)$ and $W(u ; \delta, x, y)$ for $\left\{U_{t}\right\}$ in the same way.

Risk models with a dividend barrier have been studied by many authors. We refer to Lin et al (2003) and Gerber and Shiu (2004) for surveys on the previous work. In particular, the distribution of ruin time for a model with a constant dividend barrier was discussed in Gerber (1979). In Lin et al (2003) the Gerber-Shiu discounted penalty function was further considered for the classical risk model with a constant barrier. More precisely, for $\delta>0$ the discounted penalty function can be written as

$$
m_{b, w}(u):=\mathbb{E}\left[e^{-\delta T^{*}(u)} w\left(U_{T^{*}(u)-}^{*},-U_{T^{*}(u)}^{*}\right)\right]
$$


where $w$ is a positive function. By solving the corresponding integro-differential equations, expressions of $m_{b, w}(u)$ were obtained for certain choices of $w$. Work on renewal risk models with a constant dividend barrier can be found in Li and Garrido (2004).

The optimal dividend problems have been addressed by several authors. See Gerber and Shiu (1998) for an earlier work, and Dickson and Waters (2004) for a recent work on the classical risk model. Also see Gerber and Shiu (2004) for work on a Brownian risk model.

A similar model (risk model with a two-step premium rate) was studied in Zhou (2003). In that model the surplus process is also define by (1.1), but with a premium function

$$
c(x):= \begin{cases}c_{1}, & \text { for } r \leq b, \\ c_{2}, & \text { for } r>b,\end{cases}
$$

where $c_{i}>\lambda \mu, i=1,2$. The joint distribution at ruin was obtained in Zhou (2003) for such a model.

Although the above-mentioned model with a two-step premium rate does not exactly include the risk model with a dividend barrier considered in this paper, nevertheless, the approach there can be implemented under the current setting. The key in analyzing such a model is to know when $R$ first attains level $b$ before ruin. Write

$$
T(u, b):=\inf \left\{0 \leq t<T(u): U_{t}=b\right\}
$$

with the convention that $\inf \emptyset=\infty . T(u, b)$ is then the time when dividend is first paid. Put

$$
W(u ; \delta, y) d y:=\mathbb{E}\left[e^{-\delta T(u)} ; T(u)<\infty,-U_{T(u)} \in d y\right]
$$

Let $\rho(\delta)$ and $\bar{\rho}(\delta)$ be the nonnegative and the negative solutions to

$$
c t+\lambda(\hat{p}(t)-1)=\delta, \delta \geq 0,
$$

where $\hat{p}$ denotes the Laplace transform of $p$.

Expressions for the Laplace transform of $T(u, b)$ have been obtained before. In Gerber and Shiu (1998) it was shown that, under the condition of positive safety loading $c>\lambda \mu$,

$$
\mathbb{E}\left[e^{-\delta T(u, b)}\right]=\frac{e^{\rho(\delta) u}-\psi(u)}{e^{\rho(\delta) b}-\psi(b)}
$$


In Zhou (2004) the following formula was found.

$$
\mathbb{E}\left[e^{-\delta T(u, b)}\right]=\frac{e^{\rho(\delta) u}-\int_{0}^{\infty} W(u ; \delta, y) e^{-\rho(\delta) y} d y}{e^{\rho(\delta) b}-\int_{0}^{\infty} W(b ; \delta, y) e^{-\rho(\delta) y} d y} .
$$

In fact, a formula similar to (1.4) even holds for a risk model perturbed by a Brownian motion. Its proof is an application of strong Markov property. We also want to point out that the positive safety loading condition is not necessary in obtaining (1.4).

The joint distribution of the ruin time, the surplus immediately before ruin and the deficit at ruin have been studied intensively, often under the condition of positive safety loading, since the celebrated work of Gerber and Shiu (1997). More precisely, with $c>\lambda \mu$, combining (2.8), (3.13) and (5.1) in Gerber and Shiu (1997) we have

$$
W(u ; \delta, x, y):= \begin{cases}\frac{\lambda p(x+y)\left(e^{-\rho(\delta)(x-u)}-e^{-\rho(\delta) x} \psi(u)\right)}{c(1-\psi(0))}, & \text { for } x>u, \\ \frac{\lambda p(x+y)\left(\psi(u-x)-e^{-\rho(\delta) x} \psi(u)\right)}{c(1-\psi(0))}, & \text { for } 0<x \leq u .\end{cases}
$$

Work in this line can also be found in Chiu and Yin (2003), in Wu et al (2003), and in Zhang and Wang (2003). We will add one more expression for $W(u ; \delta, x, y)$ in this paper.

Observe that the distribution of $c t-\sum_{i=1}^{N(t)} X_{i}$ has a point mass at $x=c t$, and it has a density function (denoted by $g(x, t)$ ) for $x \neq c t$. For $x \leq 0$ write $\hat{g}_{x}:=\int_{0}^{\infty} e^{-\delta t} g(x, t) d t$ for the Laplace transform of $g$ on the $t$ variable. For $x>0$ write $\hat{g}_{x}:=\frac{1}{c} e^{-\frac{(\delta+\lambda) x}{c}}+\int_{\frac{x}{c}+}^{\infty} e^{-\delta t} g(x, t) d t$.

Lemma 1.1. For any $u, \delta, x, y>0$, we have

$$
W(u ; \delta, x, y)=\lambda\left(\hat{g}_{x-u}(\delta)-e^{-\rho(\delta) x} \hat{g}_{-u}(\delta)\right) p(x+y) .
$$

Proof. Write $\pi(t ; u, x) d t$ for the probability that ruin has not not occurred by time $t$ and that there is an upcrossing of the surplus process $\left\{U_{t}\right\}$ at level $x$ between time $t$ and time $t+d t$. Then it was pointed out in Gerber and Shiu (1997) that

$$
f(u, t, x, y)=\frac{\lambda}{c} p(x+y) \pi(t ; u, x), 0 \leq x<u+c t, y \geq 0,
$$

where $f(u, t, x, y) d t d x d y:=\mathbb{P}\left\{T(u) \in d t, U_{T(u)-} \in d x,-U_{T(u)} \in d y\right\}$. We have abused notions by writing $d t, d x, d y$ for both intervals and their lengths.

The following duality was used in Gerber and Shiu (1997). Flip the sample path of $U$ upside down and run it backwards. More precisely, define

$$
\tilde{U}_{s}=U_{t}-U_{(t-s)-}, 0 \leq s \leq t .
$$


Observe that the time reversal of a Poisson point process is still a Poisson point process with the same distribution. Then $\left(U_{s}, 0 \leq s \leq t\right)$ and $\left(u+\tilde{U}_{s}, 0 \leq s \leq t\right)$ have the same joint distribution. Moreover, $\pi(t ; u, x) d t$ is equal to the probability that, starting from 0 , the process $\left\{\tilde{U}_{t}\right\}$ upcrosses level $x-u$ between time $t$ and time $t+d t$, and $\left\{\tilde{U}_{t}\right\}$ never reaches level $x$ before $t$. Observe that, in the absence of claims over time period $[t, t+d t],\left\{\tilde{U}_{t}\right\}$ upcrosses level $x-u$ between times $t$ and $t+d t$ if and only if $\tilde{U}_{t} \in[x-u-c d t, x-u]$. Applying Markov property it is evident that

$$
\pi(t ; u, x) d t=\left(g(x-u, t)-\int_{0}^{t} g(-u, t-s) d H(s)\right) c d t, 0 \leq x-u<c t,
$$

where $H$ is the distribution function of $\tilde{T}(x):=\inf \left\{t \geq 0: \tilde{U}_{t}=x\right\}$.

It is known that

$$
\mathbb{E}\left[e^{-\delta \tilde{T}(x)}\right]=e^{-\rho(\delta) x} .
$$

We first consider the case $x>u$. Notice that $H(s)=0$ for $s<\frac{x}{c}$. Taking Laplace transforms on both sides of (1.8), by (1.9) we obtain that

$$
\begin{aligned}
\int_{\frac{x-u}{c}+}^{\infty} e^{-\delta t} \pi(t ; u, x) d t & =c\left(\int_{\frac{x-u}{c}+}^{\infty} e^{-\delta t} g(x-u, t) d t-\int_{\frac{x-u}{c}+}^{\infty} e^{-\delta t} d t \int_{0}^{t} g(-u, t-s) d H(s)\right) \\
& =c\left(\int_{\frac{x-u}{c}+}^{\infty} e^{-\delta t} g(x-u, t) d t-e^{-\rho(\delta) x} \hat{g}_{-u}(\delta)\right) .
\end{aligned}
$$

In addition,

$$
\begin{aligned}
\mathbb{P} & \left\{T(u) \in\left[\frac{x-u}{c}, \frac{x-u}{c}+\frac{d x}{c}\right], U_{T(u)-} \in[x, x+d x],-U_{T(u)} \in[y, y+d y]\right\} \\
& =e^{-\lambda \frac{x-u}{c}} \frac{\lambda d x}{c} p(x+y) d y .
\end{aligned}
$$

Therefore, (1.7) implies that

$$
\begin{aligned}
W(u ; \delta, x, y) & =\frac{\lambda}{c} e^{-\frac{(\delta+\lambda)(x-u)}{c}} p(x+y)+\int_{\frac{x-u}{c}+}^{\infty} e^{-\delta t} f(u, t, x, y) d t \\
& =\lambda\left(\hat{g}_{x-u}(\delta)-e^{-\rho(\delta) x} \hat{g}_{-u}(\delta)\right) p(x+y) .
\end{aligned}
$$

Similarly, we can show that (1.6) also holds for $x \leq u$. 
Remark 1.2. The expressions for $\pi$ were also obtained in Wu et al (2003) and in Zhang and Wang (2003) for perturbed risk models.

The rest of this paper is arranged as follows. In Section 2 we first find an expression for $W^{*}(u ; \delta, x, y)$. Since

$$
m_{b, w}(u)=\int_{0}^{b} d x \int_{0}^{\infty} d y w(x, y) W^{*}(u ; \delta, x, y)+\int_{0}^{\infty} d y w(b, y) W^{*}(u ; \delta, b, y),
$$

an expression of $m_{b, w}(u)$ follows readily for any $w$. In this way we generalize the results in Lin et al (2003) to all the possible choices of $w$. The proof is an adaption of that in Zhou (2003), which involves intensive applications of Markov property.

This approach is different from that used in Lin et al (2003) and some other related work which relies on solving certain integro-differential equations. One advantage of our approach is that it is closely connected to the previous work on Gerber-Shiu functions for the classical risk models. It also goes around some technical issues like the differentiability when dealing with differential equations.

The distribution is also found in Section 2 for the last time when dividend is paid before ruin. In Section 3 we discuss the distribution of the durations for dividend payments. In particular, a new formula is derived for the expected present value of the total amount of dividend payments until ruin. Explicit results are obtained for a model with exponential claims in Section 4.

\section{JOINT DISTRIBUTION AT RUIN}

Put

$$
\bar{W}(u ; \delta, x, y) d x d y:=\mathbb{E}\left[e^{-\delta T(u)} ; T(u, b)=\infty, U_{T(u)-} \in d x, U_{T(u)} \in d y\right] .
$$

Apply strong Markov property at $T(u, b)$, we have

$$
\begin{aligned}
& \bar{W}(u ; \delta, x, y) d x d y \\
& =\mathbb{E}\left\{e^{-\delta T(u)} ; T(u, b)=\infty, U_{T(u)-} \in d x, U_{T(u)} \in d y\right\} \\
& =\mathbb{E}\left\{e^{-\delta T(u)} ; U_{T(u)-} \in d x, U_{T(u)} \in d y\right\}-\mathbb{E}\left[e^{-\delta T(u)} ; T(u, b)<\infty, U_{T(u)-} \in d x, U_{T(u)} \in d y\right] \\
& =W(u ; \delta, x, y) d x d y-\mathbb{E}\left[e^{-\delta T(u, b)}\right] \mathbb{E}\left[e^{-\delta T(b)} ; U_{T(b)-} \in d x, U_{T(b)} \in d y\right] \\
& =W(u ; \delta, x, y) d x d y-\mathbb{E}\left[e^{-\delta T(u, b)}\right] W(b ; \delta, x, y) d x d y .
\end{aligned}
$$


Therefore,

$$
\bar{W}(u ; \delta, x, y)=W(u ; \delta, x, y)-\mathbb{E}\left[e^{-\delta T(u, b)}\right] W(b ; \delta, x, y)
$$

Theorem 2.1. Given $0 \leq u<b, 0<x<b$ and $y>0$, we have

$$
\begin{aligned}
W^{*}(b ; \delta, b, y) & =\frac{\lambda p(b+y)}{\delta+\lambda-\lambda \int_{0}^{b} p(z) \mathbb{E}\left[e^{-\delta T(b-z, b)}\right] d z}, \\
W^{*}(b ; \delta, x, y) & =\frac{\lambda \int_{0}^{b} p(z) \bar{W}(b-z ; \delta, x, y) d z}{\delta+\lambda-\lambda \int_{0}^{b} p(z) \mathbb{E}\left[e^{-\delta T(b-z, b)}\right] d z},
\end{aligned}
$$

and

$$
W^{*}(u ; \delta, x, y)=\bar{W}(u ; \delta, x, y)+\mathbb{E}\left[e^{-\delta T(u ; b)}\right] W^{*}(b ; \delta, x, y)
$$

Proof. Starting from level $b$ the surplus process $\left\{U_{t}^{*}\right\}$ spends an exponential time at level $b$ until the first claim arrives. For the event $\left\{U_{T^{*}(u)-}^{*}=b, U_{T^{*}(u)}^{*} \in d y\right\}$ to occur either that claim causes ruin, or $\left\{U_{t}^{*}\right\}$ first jumps to between 0 and $b$, then comes back to level $b$ before ruin and starts all over again. Apply Markov property at the jumping time,

$$
W^{*}(b ; \delta, b, y)=\frac{\lambda}{\lambda+\delta}\left(p(b+y)+W^{*}(b ; \delta, b, y) \int_{0}^{b} p(z) \mathbb{E}\left[e^{-\delta T(b-z, b)}\right] d z\right) .
$$

Similarly, starting from $b$, for the event $\left\{U_{T^{*}(u)_{-}}^{*} \in d x, U_{T^{*}(u)}^{*} \in d y\right\}$ to occur the first claim can not cause ruin. Consequently, $\left\{U_{t}^{*}\right\}$ first jumps to somewhere between 0 and $\left\{U_{t}^{*}\right\}$. Then either ruin occurs before $\left\{U_{t}^{*}\right\}$ comes back to level $b$, or $\left\{U_{t}^{*}\right\}$ comes back to level $b$ and starts all over again. It follows that

$$
W^{*}(b ; \delta, x, y)=\frac{\lambda}{\lambda+\delta}\left(\int_{0}^{b} p(z) \bar{W}(b-z ; \delta, x, y) d z+W^{*}(b ; \delta, x, y) \int_{0}^{b} p(z) \mathbb{E}\left[e^{-\delta T(b-z, b)}\right] d z\right) .
$$

Then (2.2) and (2.3) are obtained by solving the respective equation.

Starting from $u$ either ruin occurs before $\left\{U_{t}^{*}\right\}$ ever reaches level $b$, or $\left\{U_{t}^{*}\right\}$ reaches $b$ before ruin. (2.4) also follows from strong Markov property.

Write $W(u ; x, y), W^{*}(u ; x, y)$ and $\bar{W}(u ; x, y)$ for $W(u ; 0, x, y), W^{*}(u ; 0, x, y)$ and $\bar{W}(u ; 0, x, y)$ respectively. With positive safety loading, by (1.5) we obtain

$$
W(u ; x, y):= \begin{cases}\frac{\lambda p(x+y)(1-\psi(u))}{c(1-\psi(0))}, & \text { for } x>u, \\ \frac{\lambda p(x+y)(\psi(u-x)-\psi(u))}{c(1-\psi(0))}, & \text { for } 0<x \leq u .\end{cases}
$$


More general expressions for $W(u ; x, y)$ in terms of $p$ and $\psi$ for a classical model with either positive, negative or zero safety loading can be found in Schmidli (1999).

Observe that with positive safety loading, we have

$$
\lim _{\delta \rightarrow 0+} \mathbb{E}\left[e^{-\delta T(u, b)}\right]=\mathbb{P}\{T(u, b)<\infty\}=\frac{1-\psi(u)}{1-\psi(b)},
$$

(see, eg., Proposition 7.1.10 in Asmussen (2000)). Moreover,

$$
\begin{aligned}
\bar{W}(u ; x, y) & =W(u ; x, y)-\mathbb{P}\{T(u, b)<\infty\} W(b ; x, y) \\
& =W(u ; x, y)-\frac{1-\psi(u)}{1-\psi(b)} W(b ; x, y) .
\end{aligned}
$$

We can then obtain the joint distribution of $\left(U_{T^{*}(u)-}^{*}, U_{T^{*}(u)}^{*}\right)$ by letting $\delta \rightarrow 0+$ in Theorem 2.1. Similar results were obtained in Zhou (2004) for a risk model with variable premium rate.

Proposition 2.2. Given $0 \leq u<b, 0<x<b$ and $y>0$, with positive safety loading, we have

$$
\begin{gathered}
W^{*}(b ; b, y)=\frac{\lambda p(b+y)(1-\psi(b))}{\lambda(1-\psi(b))-\lambda \int_{0}^{b} p(z)(1-\psi(b-z)) d z}, \\
W^{*}(b ; x, y)=\frac{\lambda \int_{0}^{b} p(z)[(1-\psi(b)) W(b-z ; x, y)-(1-\psi(b-z)) W(b ; x, y)] d z}{\lambda(1-\psi(b))-\lambda \int_{0}^{b} p(z)(1-\psi(b-z)) d z},
\end{gathered}
$$

and

$$
W^{*}(u ; x, y)=W(u ; x, y)-\frac{1-\psi(u)}{1-\psi(b)} W(b ; x, y)+\frac{1-\psi(u)}{1-\psi(b)} W^{*}(b ; x, y) .
$$

We can also find an expression of the Laplace transform of $T^{*}(u)$.

Proposition 2.3. Given $0 \leq u<b$, we have

$$
\mathbb{E}\left[e^{-\delta T^{*}(b)}\right]=\frac{\lambda(1-P(b))+\lambda \int_{0}^{b} p(z)\left(\mathbb{E}\left[e^{-\delta T(b-z)}\right]-\mathbb{E}\left[e^{-\delta T(b-z, b)}\right] \mathbb{E}\left[e^{-\delta T(b)}\right]\right) d z}{\delta+\lambda-\lambda \int_{0}^{b} p(z) \mathbb{E}\left[e^{-\delta T(b-z, b)}\right] d z}
$$

and

$$
\mathbb{E}\left[e^{-\delta T^{*}(u)}\right]=\mathbb{E}\left[e^{-\delta T(u)}\right]-\mathbb{E}\left[e^{-\delta T(u, b)}\right] \mathbb{E}\left[e^{-\delta T(b)}\right]+\mathbb{E}\left[e^{-\delta T(u, b)}\right] \mathbb{E}\left[e^{-\delta T^{*}(b)}\right] .
$$

Proof. Take integrals on both sides of (2.2), (2.3) and (2.4). Recycling the proof in Theorem 2.1 we see that

$$
\left.\int_{0}^{b-} d x \int_{0}^{\infty} W(b-z ; \delta, x, y)\right) d y=\mathbb{E}\left[e^{-\delta T(b-z)}\right]-\mathbb{E}\left[e^{-\delta T(b-z, b)}\right] \mathbb{E}\left[e^{-\delta T(b)}\right] .
$$

Then (2.6) and (2.7) follow. 
Our last result in this section concerns the last time when a dividend is paid before ruin. Set

$$
T^{\prime}(u, b):=\sup \left\{0 \leq t \leq T(u): R_{t}=b\right\}
$$

with convention that $\sup \{\emptyset\}=0$.

Theorem 2.4. For $0 \leq u \leq b$, we have

$$
\mathbb{E}\left[e^{-\delta T^{\prime}(u, b)}\right]=\frac{\lambda(1-P(b)) \mathbb{E}\left[e^{-\delta T(u, b)}\right]}{\lambda+\delta-\lambda \int_{0}^{b} p(z) \mathbb{E}\left[e^{-\delta T(b-z, b)}\right] d z}
$$

Proof. Starting from $b$, the next claim arrives after an exponential time. Conditioning on the size of that claim we have that

$$
\mathbb{E}\left[e^{-\delta T^{\prime}(b, b)}\right]=\frac{\lambda}{\lambda+\delta}\left(1-P(b)+\mathbb{E}\left[e^{-\delta T^{\prime}(b, b)}\right] \int_{0}^{b} p(z) \mathbb{E}\left[e^{-\delta T(b-z, b)}\right] d z\right) .
$$

Moreover, $T^{\prime}(u, b) \geq T(u, b)$ if and only if $T(u, b)<\infty$. An application of strong Markov property at time $T(u, b)$ gives

$$
\mathbb{E}\left[e^{-\delta T^{\prime}(u, b)}\right]=\mathbb{E}\left[e^{-\delta T(u, b)}\right] \mathbb{E}\left[e^{-\delta T^{\prime}(b, b)}\right] .
$$

The assertion of this theorem thus follows.

\section{Present value of Dividends until Ruin}

Write $R_{i}$ (resp., $L_{i}$ ) for the time when $\left\{U_{t}^{*}\right\}$ reaches (resp., leaves) level $b$ from below (resp., above) for the $i$-th time. Then $0 \leq R_{1}<L_{1}<R_{2}<L_{2}<\ldots$, and $L_{i}-R_{i}$ represents the duration of the $i$-th period of premium payment. The Laplace transform of $R_{1}$ is given by either (1.3) or (1.4). Let $M:=\sup \left\{i: R_{i}<T(u)\right\}$ with $\sup \emptyset:=0 . M$ then stands for the total number of premium payment periods before ruin.

Write $p_{0}:=\mathbb{P}\left\{R_{1}<\infty\right\}$ and $p_{1}:=\mathbb{P}\left\{R_{2}<\infty \mid R_{1}<\infty\right\}$. With positive safety loading it is easy to see that

$$
p_{0}=\mathbb{P}\{T(u, b)<\infty\}=\frac{1-\psi(u)}{1-\psi(b)} .
$$

Starting from level $b$, after the first downward jump the overall probability of ruin before $\left\{U_{t}^{*}\right\}$ ever climbs back to $b$ again is

$$
1-P(b)+\int_{0}^{b} p(z) \mathbb{P}\{T(b-z, b)=\infty\} d z=1-P(b)+\int_{0}^{b} p(z) \frac{\psi(b-z)-\psi(b)}{1-\psi(b)} d z .
$$


So,

$$
p_{1}:=\int_{0}^{b} p(z) \frac{1-\psi(b-z)}{1-\psi(b)} d z
$$

Our next result is a consequence of strong Markov property for $\left\{U_{t}^{*}\right\}$.

Proposition 3.1. $\mathbb{P}\{M=0\}=1-p_{0}$ and $\mathbb{P}\{M=k\}=p_{0}\left(1-p_{1}\right) p_{1}^{k-1}, k \geq 1$. Given $M=k$, $\left\{L_{i}-R_{i}, i=1, \ldots, k\right\}$ are $i . i . d$. exponential random variables with mean $\lambda^{-1} .\left\{R_{i+1}-L_{i}, i=\right.$ $1, \ldots, k-1\}$ are i.i.d. random variables with a common Laplace transform

$$
\mathbb{E}\left[e^{-\delta\left(R_{2}-L_{1}\right)} \mid M=k\right]=\frac{1}{p_{1}} \int_{0}^{b} p(y) \mathbb{E}\left[e^{-\delta T(b-y, b)}\right] d y, \delta \geq 0 .
$$

The two sequences are also independent.

Remark 3.2. It follows from Proposition 3.1 that the total dividend time $\sum_{i=1}^{M}\left(L_{i}-R_{i}\right)$ is a (defective) geometric summation of i.i.d. exponential random variables. A somewhat similar work on duration of the time in red for the classical risk model can be found in Dos Reis (1993) and in Dickson and Dos Reis (1996).

Let $\delta \geq 0$ be the force of interest for valuation. The present value of the total amount of dividend payments until ruin is

$$
D_{\delta}(u, b):=1_{\{M \geq 1\}} \sum_{i=1}^{M} \int_{R_{i}}^{L_{i}} c e^{-\delta t} d t=\frac{c}{\delta} 1_{\{M \geq 1\}} \sum_{i=1}^{M}\left(e^{-\delta R_{i}}-e^{-\delta L_{i}}\right) .
$$

Theorem 3.1 gives an explicit description on the distribution of $D_{\delta}(u, b)$. It allows us to carry out some detailed computations. The next theorem concerns the expected value of $D_{\delta}(u, b)$.

Theorem 3.3. For $0 \leq u \leq b$ and $\delta \geq 0$, we have

$$
\mathbb{E}\left[D_{\delta}(u, b)\right]=\frac{c \mathbb{E}\left[e^{-\delta T(u, b)}\right]}{\lambda+\delta-\lambda \int_{0}^{b} p(y) \mathbb{E}\left[e^{-\delta T(b-y, b)}\right] d y} .
$$


Proof. By Proposition 3.1, we obtain

$$
\begin{aligned}
& \mathbb{E}\left[D_{\delta}(u, b)\right] \\
& =\frac{c}{\delta} \mathbb{E}\left[\sum_{m=1}^{\infty} \sum_{i=1}^{m}\left(e^{-\delta\left(R_{1}+\sum_{j=1}^{i-1}\left\{\left(L_{j}-R_{j}\right)+\left(R_{j+1}-L_{j}\right)\right\}\right)}\right) \mid M=m\right] \mathbb{P}\{M=m\} \\
& -\frac{c}{\delta} \mathbb{E}\left[\sum_{m=1}^{\infty} \sum_{i=1}^{m}\left(e^{-\delta\left(R_{1}+\sum_{j=1}^{i-1}\left\{\left(L_{j}-R_{j}\right)+\left(R_{j+1}-L_{j}\right)\right\}+\left(L_{i}-R_{i}\right)\right)}\right) \mid M=m\right] \mathbb{P}\{M=m\} \\
& =\frac{c\left(1-p_{1}\right)}{\delta} \mathbb{E}\left[e^{-\delta T(u, b)}\right] \sum_{m=0}^{\infty} p_{1}^{m} \sum_{i=0}^{m}\left(\frac{\lambda}{(\lambda+\delta) p_{1}} \int_{0}^{b} p(y) \mathbb{E}\left[e^{-\delta T(b-y, b)}\right] d y\right)^{i} \\
& -\frac{c\left(1-p_{1}\right)}{\delta} \mathbb{E}\left[e^{-\delta T(u, b)}\right] \sum_{m=0}^{\infty} p_{1}^{m} \sum_{i=0}^{m} \frac{\lambda}{\lambda+\delta}\left(\frac{\lambda}{(\lambda+\delta) p_{1}} \int_{0}^{b} p(y) \mathbb{E}\left[e^{-\delta T(b-y, b)}\right] d y\right)^{i} \\
& =\frac{c\left(1-p_{1}\right) \mathbb{E}\left[e^{-\delta T(u, b)}\right]}{\lambda+\delta} \sum_{m=0}^{\infty} p_{1}^{m} \sum_{i=0}^{m}\left(\frac{\lambda}{(\lambda+\delta) p_{1}} \int_{0}^{b} p(y) \mathbb{E}\left[e^{-\delta T(b-y, b)}\right] d y\right)^{i} \\
& =\frac{c\left(1-p_{1}\right) \mathbb{E}\left[e^{-\delta T(u, b)}\right]}{\lambda+\delta} \frac{\sum_{m=0}^{\infty}\left[p_{1}^{m+1}-\left(\frac{\lambda}{\lambda+\delta} \int_{0}^{b} p(y) \mathbb{E}\left[e^{-\delta T(b-y, b)}\right] d y\right)^{m+1}\right]}{p_{1}-\frac{\lambda}{\lambda+\delta} \int_{0}^{b} p(y) \mathbb{E}\left[e^{-\delta T(b-y, b)}\right] d y} \\
& =\frac{c\left(1-p_{1}\right) \mathbb{E}\left[e^{-\delta T(u, b)}\right]}{(\lambda+\delta)\left(p_{1}-\frac{\lambda}{\lambda+\delta} \int_{0}^{b} p(y) \mathbb{E}\left[e^{-\delta T(b-y, b)}\right] d y\right)}\left(\frac{p_{1}}{1-p_{1}}-\frac{\frac{\lambda}{\lambda+\delta} \int_{0}^{b} p(y) \mathbb{E}\left[e^{-\delta T(b-y, b)}\right] d y}{1-\frac{\lambda}{\lambda+\delta} \int_{0}^{b} p(y) \mathbb{E}\left[e^{-\delta T(b-y, b)}\right] d y}\right) \\
& =\frac{c \mathbb{E}\left[e^{-\delta T(u, b)}\right]}{\lambda+\delta-\lambda \int_{0}^{b} p(y) \mathbb{E}\left[e^{-\delta T(b-y, b)}\right] d y} .
\end{aligned}
$$

Remark 3.4. It was shown in Gerber and Shiu (1998) that, with positive safety loading,

$$
\mathbb{E}\left[D_{\delta}(u, b)\right]=\frac{e^{\rho(\delta) u}-\psi(u)}{\rho(\delta) e^{\rho(\delta) b}-\psi^{\prime}(b)},
$$

where $\psi^{\prime}$ is the derivative of $\psi$. One can compare (3.2) with (3.1). It would be interesting if one can find a simple way to reconcile these two results.

By an optimal dividend barrier we mean a value $b^{*} \geq u$ such that

$$
\mathbb{E}\left[D_{\delta}\left(u, b^{*}\right)\right]=\sup _{b \geq u} \mathbb{E}\left[D_{\delta}(u, b)\right]
$$


Remark 3.5. Set $\delta=0$ in (3.1),

$$
\begin{aligned}
\mathbb{E}\left[D_{0}(u, b)\right] & =\frac{c \mathbb{P}\{T(u, b)<\infty\}}{\lambda-\lambda \int_{0}^{b} p(y) \mathbb{P}\{T(b-y, b)<\infty\} d y} \\
& =\frac{c(1-\psi(u))}{\lambda\left(1-P(b)-\psi(b)+\int_{0}^{b} p(z) \psi(b-z) d z\right)} .
\end{aligned}
$$

It is not hard to see that with positive safety loading,

$$
\lim _{b \rightarrow \infty} \int_{0}^{b} p(z) \psi(b-z) d z=0 .
$$

Then

$$
\lim _{b \rightarrow \infty} \mathbb{E}\left[D_{0}(u, b)\right]=\infty .
$$

So, there is no meaningful optimal dividend barrier in this situation.

On the other hand, for $\delta>0$, we have

$$
\lim _{b \rightarrow \infty} \mathbb{E}\left[e^{-\delta T(u, b)}\right]=0 .
$$

Then it follows form Theorem 3.3 that

$$
\lim _{b \rightarrow \infty} \mathbb{E}\left[D_{\delta}(u, b)\right]=0
$$

So, the optimal dividend barrier always exists for $\delta>0$.

\section{An eXAmple}

In this section we consider a model in which the claim size $U_{i}$ follows an exponential distribution with mean $\beta^{-1}$. We assume that $c>\lambda \beta^{-1}$. Then $p(x)=\beta e^{-\beta x}$ and $\hat{p}(s)=\frac{\beta}{\beta+s}$.

Equation (1.2) has a positive solution

$$
\rho(\delta)=\frac{\lambda+\delta-c \beta+\sqrt{(c \beta-\lambda-\delta)^{2}+4 c \beta \delta}}{2 c}
$$

and a negative solution

$$
\bar{\rho}(\delta)=\frac{\lambda+\delta-c \beta-\sqrt{(c \beta-\lambda-\delta)^{2}+4 c \beta \delta}}{2 c} .
$$

It is known that $\psi(u)=\frac{\lambda}{c \beta} e^{\frac{u(\lambda-c \beta)}{c}}$ and $\mathbb{E}\left[e^{-\delta T(u)}\right]=e^{\bar{\rho}(\delta) u}\left(1+\frac{\bar{\rho}(\delta)}{\beta}\right)$. See Proposition 1.2 in Chapter IV of Asmussen (2000). 
It follows from Gerber and Shiu (1998) or Zhou (2004) that

$$
\mathbb{E}\left[e^{-\delta T(u, v)}\right]=\frac{(\beta+\rho(\delta)) e^{\rho(\delta) u}-(\beta+\bar{\rho}(\delta)) e^{\bar{\rho}(\delta) u}}{(\beta+\rho(\delta)) e^{\rho(\delta) v}-(\beta+\bar{\rho}(\delta)) e^{\bar{\rho}(\delta) v}} .
$$

By (1.5) we can find a formula for $W(u ; \delta, x, y)$.

$$
W(u ; \delta, x, y):= \begin{cases}\frac{\lambda \beta e^{-\beta(x+y)}\left(c \beta e^{-\rho(\delta)(x-u)}-\lambda e^{-\rho(\delta) x+u(\lambda-c \beta) / c}\right)}{c(c \beta-\lambda)}, & \text { for } x>u, \\ \frac{\lambda^{2} \beta e^{-\beta(x+y)}\left(e^{(u-x)(\lambda-c \beta) / c}-e^{-\rho(\delta) x+u(\lambda-c \beta) / c}\right)}{c(c \beta-\lambda)}, & \text { for } 0<x \leq u .\end{cases}
$$

Then by (2.1) we can reach an expression for $\bar{W}(u ; \delta, x, y)$, which leads to explicit, but rather complicated, expressions for $W^{*}(u ; \delta, x, y)$ by Theorem 2.1 .

The expression of $\mathbb{E}\left[e^{-\delta T^{*}(u)}\right]$ is simpler. Since

$$
\begin{aligned}
\int_{0}^{b} p(z) \mathbb{E}\left[e^{-\delta T(b-z, b)}\right] d z & =\int_{0}^{b} \beta e^{-\beta z} \frac{(\beta+\rho(\delta)) e^{\rho(\delta)(b-z)}-(\beta+\bar{\rho}(\delta)) e^{\bar{\rho}(\delta)(b-z)}}{(\beta+\rho(\delta)) e^{\rho(\delta) b}-(\beta+\bar{\rho}(\delta)) e^{\bar{\rho}(\delta) b}} d z \\
& =\frac{\beta e^{\rho(\delta) b}-\beta e^{\bar{\rho}(\delta) b}}{(\beta+\rho(\delta)) e^{\rho(\delta) b}-(\beta+\bar{\rho}(\delta)) e^{\bar{\rho}(\delta) b}}
\end{aligned}
$$

and

$$
\begin{aligned}
\int_{0}^{b} p(z) \mathbb{E}\left[e^{-\delta T(b-z)}\right] d z & =\int_{0}^{b} \beta e^{-\beta z} e^{\bar{\rho}(\delta)(b-z)}\left(1+\frac{\bar{\rho}(\delta)}{\beta}\right) d z \\
& =e^{\bar{\rho}(\delta) b}-e^{-\beta b} .
\end{aligned}
$$

Then by $(2.6)$

$$
\begin{aligned}
\mathbb{E}\left[e^{-\delta T^{*}(b)}\right] & =\frac{\lambda\left\{e^{-\beta b}+e^{\bar{\rho}(\delta) b}-e^{-\beta b}-e^{\bar{\rho}(\delta) b}\left(1+\frac{\bar{\rho}(\delta)}{\beta}\right) \frac{\beta e^{\rho(\delta) b}-\beta e^{\bar{\rho}(\delta) b}}{(\beta+\rho(\delta)) e^{\rho(\delta) b}-(\beta+\bar{\rho}(\delta)) e^{\bar{\rho}(\delta) b}}\right\}}{\delta+\lambda-\lambda \frac{\beta e^{\rho(\delta) b}-\beta e^{\bar{\rho}(\delta) b}}{(\beta+\rho(\delta)) e^{\rho(\delta) b}-(\beta+\bar{\rho}(\delta)) e^{\bar{\rho}(\delta) b}}} \\
& =\frac{\lambda(\rho(\delta)-\bar{\rho}(\delta)) e^{(\rho(\delta)+\bar{\rho}(\delta)) b}}{(\delta \beta+(\delta+\lambda) \rho(\delta)) e^{\rho(\delta) b}-(\delta \beta+(\delta+\lambda) \bar{\rho}(\delta)) e^{\bar{\rho}(\delta) b}} .
\end{aligned}
$$

Hence, $\mathbb{E}\left[e^{-\delta T^{*}(u)}\right], 0 \leq u \leq b$, follows readily.

By Theorem 2.4, we have

$$
\mathbb{E}\left[e^{-\delta T^{\prime}(u, b)}\right]=\frac{\lambda \beta e^{-\beta(b+y)}\left[(\beta+\rho(\delta)) e^{\rho(\delta) b}-(\beta+\bar{\rho}(\delta)) e^{\bar{\rho}(\delta) b}\right]}{[\delta \beta+(\lambda+\delta) \rho(\delta)] e^{\rho(\delta) b}-[\delta \beta+(\lambda+\delta) \bar{\rho}(\delta)] e^{\bar{\rho}(\delta) b}}
$$


By Theorem 3.3,

$$
\begin{aligned}
& \mathbb{E}\left[D_{\delta}(u, b)\right] \\
& =\frac{c \frac{(\beta+\rho(\delta)) e^{\rho(\delta) u}-(\beta+\bar{\rho}(\delta)) e^{\bar{\rho}(\delta) u}}{(\beta+\rho(\delta)) e^{\rho(\delta) b}-(\beta+\bar{\rho}(\delta)) e^{\bar{\rho}(\delta) b}}}{\lambda+\delta-\lambda \int_{0}^{b} \beta e^{-\beta y \frac{(\beta+\rho(\delta)) e^{\rho(\delta)(b-y)}-(\beta+\bar{\rho}(\delta)) e^{\bar{\rho}(\delta)(b-u)}}{(\beta+\rho(\delta)) e^{\rho(\delta) b}-(\beta+\bar{\rho}(\delta)) e^{\bar{\rho}(\delta) b}} d y}} \\
& =\frac{c\left[(\beta+\rho(\delta)) e^{\rho(\delta) u}-(\beta+\bar{\rho}(\delta)) e^{\bar{\rho}(\delta) u}\right]}{(\lambda+\delta)\left[(\beta+\rho(\delta)) e^{\rho(\delta) b}-(\beta+\bar{\rho}(\delta)) e^{\bar{\rho}(\delta) b]}-\lambda \beta\left(e^{\rho(\delta) b}-e^{\bar{\rho}(\delta) b)}\right.\right.} \\
& =\frac{c\left[(\beta+\rho(\delta)) e^{\rho(\delta) u}-(\beta+\bar{\rho}(\delta)) e^{\bar{\rho}(\delta) u}\right]}{[\delta \beta+(\lambda+\delta) \rho(\delta)] e^{\rho(\delta) b}-[\delta \beta+(\lambda+\delta) \bar{\rho}(\delta)] e^{\bar{\rho}(\delta) b}} .
\end{aligned}
$$

With some algebra it is easy to check that (4.1) is exactly the same as (7.8) in Gerber and Shiu (1998).

Acknowledgement The author thanks Elias Shiu and an anonymous referee for very helpful comments.

\section{REFERENCES}

[1] Asmussen, S., 2000. Ruin probabilities. Word Scientific.

[2] Chiu, S., Yin, C., 2003. The time of ruin, the surplus prior to ruin and the deficit at ruin for the classical risk process perturbed by diffusion. Insurance: Mathematics and Economics 33, 59-66.

[3] Dickson, D.C.M., Dos Reis, A.D.E., 1996. On the distribution of duration of negative surplus. Scandinavian Actuarial Journal 2, 148-164.

[4] Dickson D.C.M., Waters H.R., 2004. Some optimal dividends problems. Astin Bulletin 34, 49-74.

[5] Dos Reis, A.D.E., 1993. How long is the surplus below zero? Insurance: Mathematics and Economics 12, $23-38$.

[6] Gerber, H.U., 1979. An introduction to mathematical risk theory. S.S. Huebner Foundation Monographs, University of Pennsylvania.

[7] Gerber, H.U., Shiu, E.S.W., 1997. The joint distribution of the time of ruin, the surplus immediately before ruin, and the deficit at ruin. Insurance: Mathematics and Economics 21, 129-137.

[8] Gerber, H.U., Shiu, E.S.W., 1998. On the time value of ruin. North American Actuarial Journal 2, 48-78.

[9] Gerber, H.U., Shiu, E.S.W., 2004. Optimal dividends: analysis with Brownian motion. North American Actuarial Journal 8, 1-20.

[10] Li, S., Garrido, J., 2004. On a class of renewal risk models with a constant dividend barrier. To appear in Insurance: Mathematics and Economics.

[11] Lin, X., Willmot, G.E., Drekic, S., 2003. The classical risk model with a constant dividend barrier: Analysis of the Gerber-Shiu discounted penalty function. Insurance: Mathematics and Economics 33, 551-566.

[12] Schmidli H., 1999. On the distribution of the surplus prior and at ruin. Astin Bulletin 29, $227-244$. 
[13] Wu R., Wang G., Wei L., 2003. Joint distributions of some actuarial random vectors containing the time of ruin. Insurance: Mathematics and Economics 33, 147-161.

[14] Zhang, C., Wang, G., 2003. The joint density function of three characteristics on jump-diffusion risk process. Insurance: Mathematics and Economics 32, 445-455.

[15] Zhou, X., 2003. When does surplus reach a certain level before ruin? To appear in Insurance: Mathematics and Economics.

[16] Zhou, X., 2004. Risk model with a two-step premium rate. Submitted 


\section{List of Recent Technical Reports}

46. M. L. Filshtinsky, R. Rodríguez-Ramos and O. Sanchez-Casals, Fracture Mechanic in Piezoceramic Composite Plate, June 2000

47. F. Lebon, R. Rodriguez-Ramos and A. Mesejo, Homogenization and Wavelet-Galerkin Method for a Nonlinear One-dimensional Problem, June 2000

48. L. Yang, The Impact of Mortality Improvement on Social Security, August 2000

49. Rodrigo Arias López and José Garrido, Bounds and Other Properties of the Inverse, Moments of a Positive Binomial Variate, September 2000

50. B. N. Dimitrov, Z. Khalil, M. E. Ghitany and V. V. Rykov, Likelihood Ratio Test for Almost Lack of Memory Distributions, November 2001

51. Yogendra P. Chaubey and Anthony Crisalli, The Generalized Smoothing Estimator, April 2002

52. Yogendra P. Chaubey and Pranab K. Sen, Smooth Isotonic Estimation of Density, Hazard and MRL Functions, April 2002

53. Pablo Olivares, Maximum Likelihood Estimators for a Branching-Diffusion Process, August 2002

54. Shuanming Li and José Garrido, On Ruin for the Erlang(n) Risk Process, June 2003

55. G. Jogesh Babu and Yogendra P. Chaubey, Smooth Estimation of a Distribution and Density function on a Hypercube Using Bernstein Polynomials for Dependent Random Vectors, August 2003

56. Shuanming Li and José Garrido, On the Time Value of Ruin for a Sparre Anderson Risk Process Perturbed by Diffusion, November 2003

57. Yogendra P. Chaubey, Cynthia M. DeSouza and Fassil Nebebe, Bayesian Inference for Small Area Estimation under the Inverse Gaussian Model via Cibbs Sampling, December 2003 
58. Alexander Melnikov and Victoria Skornyakova, Pricing of EquityLinked Life Insurance Contracts with Flexible Guarantees, May 2004

59. Yi Lu and José Garrido, Regime-Switching Periodic Models for Claim Counts, June 2004.

60. I. Urrutia-Romaní, R. Rodríguez-Ramos, J. Bravo-Castillero and R. Guinovart-Díaz, Asymptotic Homogenization Method Applied to Linear Viscoelastic Composites. Examples, August 2004.

61. Yi Lu and José Garrido, Double Periodic Non-Homogeneous Poisson Models for Hurricanes Data, September 2004.

62. M.I. Beg and M. Ahsanullah, On Characterizing Distributions by Conditional Expectations of Functions of Generalized Order Statistics, September, 2004.

63. M.I. Beg and M. Ahsanullah, Concomitants of Generalized Order Statistics from Farlie-Gumbel-Morgenstern Distributions, September, 2004.

64. Yogendra P. Chaubey and Debaraj Sen, An investigation into properties of an estimator of mean of an inverse Gaussian population, September, 2004.

65. Steven N. Evans and Xiaowen Zhou, Balls-in-boxes duality for coalescing random walks and coalescing Brownian motions, September, 2004.

66. Qihe Tang, Asymptotic ruin probabilities of the renewal model with constant interest force and regular variation, November, 2004.

67. Xiaowen Zhou, On a classical risk model with a constant dividend barrier, November, 2004.

Copies of technical reports can be requested from:

Prof. Xiaowen Zhou

Department of Mathematics and Statistics

Concordia University

7141, Sherbrooke Street West

Montréal (QC) H4B 1R6 CANADA 